\title{
Menschen mit geistigen und mehrfachen Behinderungen in der akut-stationären Versorgung
}

\author{
Ausgewählte Ergebnisse einer qualitativ-explorativen Untersuchung
}

\author{
M. Hasseler ${ }^{1}$
}

\section{Zusammenfassung}

Hintergrund | Nationale und internationale Publikationen weisen auf eine höhere Bedeutsamkeit von Menschen mit Behinderungen in gesundheitlicher und pflegerischer Versorgung hin. Aus der internationalen Literaturlage ist zu entnehmen, dass für diese Zielgruppe die Klinikversorgung in vielerlei Hinsicht als defizitär zu betrachten ist.

Fragestellung I Diese Studie intendiert, Erfahrungen von Mitarbeitern ambulanter und stationärer Wohneinrichtungen für Menschen mit geistigen und mehrfachen Behinderungen und Angehörige der Zielgruppe hinsichtlich der gesundheitlichen und pflegerischen Versorgung qualitativ-explorativ zu untersuchen.

Methode | Es wurden 21 leitfadengestützte Interviews mit Mitarbeitern entsprechender Institutionen, in denen die Zielgruppe versorgt und betreut wird, oder mit Angehörigen bzw. Personen des näheren sozialen Umfeldes als Stellvertreter der Zielgruppe durchgeführt.

Ergebnisse | Die Interviews wurden mit dem Softwareprogramm MAXQDA nach der GroundedTheory-Methode ausgewertet. Die Ergebnisse zeigen vor allem, dass in der Wahrnehmung und aus der Erfahrung der Befragten im Krankenhaus ein „Mangel“ besteht, z. B. an Zeit, Personal, Ressourcen, Qualifikation, Kooperation u.Ä. Mitarbeiter von Wohneinrichtungen und Angehörige der Zielgruppe müssen sich vielfach in einem hohen Maße einbringen, damit pflegerische und gesundheitliche Leistungen in akut-stationärer Versorgung erbracht werden.

Folgerung | Die Ergebnisse deuten auf eine nicht ausreichende Versorgung von Menschen mit Behinderungen im Krankenhaus hin. Es ist erforderlich, diese kritisch zu reflektieren und angemessene Maßnahmen, Konzepte und Interventionen bzw. Regelwerke zu entwickeln.

\section{Einleitung}

In den letzten Jahren geraten Fragen der gesundheitlichen und pflegerischen Versorgung von Menschen mit Behinderungen zunehmend in das Blickfeld gesundheitswissenschaftlicher, medizinischer sowie pflegewissenschaftlicher Diskussionen. In einigen Veröffentlichungen werden Menschen mit Behinderungen als eine gefährdete Gruppe im Gesundheitswesen betrachtet, weil zum einen eine Reihe von Faktoren wie

- chronische Erkrankungen,

- sekundäre Erkrankungen,

- Versorgungs- und Betreuungssituation,

- ökonomischer Status u.Ä.

die medizinische und gesundheitliche Versorgung sowie den Gesundheitszustand beeinflussen [16]. Zum anderen erscheint der Zugang zur Gesundheitsversorgung für Menschen mit Behinderungen erschwert [29, 32]. Die Literaturlage weist auf einige Defizite der gesundheitlichen und pflegerischen Versorgung von Menschen mit geistigen und mehrfachen Behinderungen in Kliniken hin. Es wird berichtet über [21, 29, 31, 28, 3]
- nicht angemessene Kommunikation mit der Zielgruppe,

- nicht angemessene therapeutische und pflegerische Versorgung,

- mangelndes Verständnis von Behinderung (vom medizinischen Personal vielfach als chronische Krankheit eingeordnet),

- nicht ausreichende Qualifikation,

- mangelndes Wissen des medizinischen Personals über die Zielgruppe sowie

- indifferente Haltung gegenüber dieser Personengruppe.

Zusammenfassend entsteht aus der Literaturlage der Eindruck, dass substanzielle Gesundheitsbedürfnisse von Menschen mit geistigen und mehrfachen Behinderungen nicht erfüllt werden [11].

Gleichwohl können die Ergebnisse nicht problemlos auf Deutschland übertragen werden. Sowohl die Finanzierung der Versorgung im Krankenhaus sowie die Zugangsvoraussetzungen für gesundheitliche Leistungen wie auch Qualifikationen, Aufgaben und Verantwortlichkeiten der Gesundheitsprofessionen sind im internationalen Raum unterschiedlich. Vor diesem Hintergrund wird eine qualitative explorative Untersuchung durch-
Institute

1 Ostfalia Hochschule für angewandte Wissenschaften, Wolfsburg

\section{Korrespondenz}

Prof. Dr. habil.

Martina Hasseler

Ostfalia Hochschule für angewandte Wissenschaften, Fakultät Gesundheitswesen, Campus Wolfsburg Rothenfelder Str. 10 38440 Wolfsburg Tel. 05361892223250 Fax. 05361892223251 m.hasseler@ostfalia.de 
geführt, die zunächst die Erfahrungen und das Erleben von Angehörigen von Menschen mit geistigen und mehrfachen Behinderungen und Mitarbeitern in den jeweiligen Einrichtungen, in denen Menschen mit geistigen und mehrfachen Behinderungen leben, zum Inhalt haben.

Demgemäß lautet unsere Fragestellung: Welche Erfahrungen machen Mitarbeiter von stationären und ambulanten Wohneinrichtungen für Menschen mit geistigen und mehrfachen Behinderungen und Angehörige der Betroffenen in der gesundheitlichen und pflegerischen Versorgung?

\section{Material und Methoden}

Als Forschungsdesign wurde die „Grounded Theory“ [13] gewählt, da in dieser explorativen Erhebung die Prozesse und Interaktionen der Beteiligten im Vordergrund stehen. Mit dieser Fragestellung sollen Erkenntnisse über die Erfahrungen der

- Betreuer

- Mitarbeiter

- Angehörigen als Stellvertreter

systematisch eruiert werden, um auf diesem Wege die gesundheitliche und pflegerische Versorgung aus der Perspektive der Betroffenen zu verstehen und theoretische Erkenntnisse zu generieren $[7,13]$.

Als Datenerhebungsmethode wurden Experteninterviews in Form von Leitfadeninterviews angewendet [1], da mit Hilfe dieser Methode Spezialwissen und Handlungsroutinen der Befragten systematisch erschlossen werden sollen [14,23]. Des Weiteren ist diese Methode besonders geeignet, wenn der Zugang zum Feld schwierig, tabuisiert oder ethisch diffizil ist $[1,23]$. Es wurden Mitarbeiter oder Eltern / Angehörige befragt, da bislang noch wenige systematische Erfahrungen in der Befragung der Zielgruppe zu so einem diffizilen Thema vorliegen. Die Literaturlage zeigt bisher, dass die Befragung von Menschen mit geistigen und mehrfachen Behinderungen selbst aus forschungsmethodischer und ethischer Sicht diffizil ist. Bislang ist noch kein angemessenes Befragungsinstrument entwickelt worden.

Die Stichprobenbildung erfolgt nach dem Prinzip des „theoretischen Samplings“ [13]. Das heißt, die Anzahl der Befragten wird nicht im Vorhinein festgelegt, sondern geleitet von der Notwendigkeit, aus dem Datenmaterial entstehende Konzepte theoretisch zu sättigen [9]. Des Weiteren ist damit impliziert, dass Datenerhebung und -auswertung (Analyse, Kodierung) parallel erfolgt [30]. Für die Findung von signifikanten Individuen bzw. Experten wurden im Vorfeld Einschlusskriterien formuliert, um Personen zu befragen, die zur interessierenden Forschungsfrage Antworten geben können und in diesem Sinne signifikant für das Projekt sind [9]:
- Beherrschung der deutschen Sprache in Sprache und im Verständnis,

- Mitarbeiter von ambulanten oder stationären Einrichtungen für Menschen mit geistigen und mehrfachen Behinderungen und

- Zuständigkeit für die gesundheitliche Versorgung oder

- Angehöriger mit entsprechender Verantwortlichkeit für einen Vertreter der Zielgruppe.

Es wurden 21 Interviews in einem Zeitraum von 3 Monaten (Juli 2013 bis September 2013) in einer Region in Norddeutschland in und um eine Universitätsstadt durchgeführt. Sie dauerten zwischen 30 und 75 Minuten. An den Interviews haben 15 Mitarbeiter und 6 Eltern teilgenommen. Das Alter der befragen Mitarbeiter lag zwischen 31 und 63 Jahren. Die meisten Befragten in dieser Stichprobe waren weiblich (11 Interviewte). 4 Befragte waren männlich. Die Mitarbeiter waren in Einrichtungen tätig, die von konfessionell getragenen Trägern (2 Einrichtungen) oder gemeinnützigen Gesellschaften geführt werden (3 Einrichtungsträger). Bei den Eltern wurden 2 Väter, 1 Mutter und 3 Elternpaare interviewt. Die Eltern übernahmen jeweils die Funktion der gesetzlichen Betreuer. Das Alter der Eltern variierte zwischen 68 und 74 Jahre. Die Kinder leben zwischen 12 und 42 Jahren in den Wohneinrichtungen.

Die Datenauswertung wurde mit MAXQDA 11 durchgeführt und orientierte sich an das Verfahren der Grounded Theory [2], welches im von Meuser und Nagel [22] skizzierten Datenauswertungsverfahren von Experteninterviews aufgenommen wird.

Es wurde kein Ethikvotum eingeholt, da keine Interventionen oder Maßnahmen bei Menschen durchgeführt wurden. Die Teilnehmer wurden mündlich und schriftlich über die Studie informiert und hatten jederzeit die Möglichkeit, die Teilnahme wieder zurückzuziehen.

\section{Ergebnisse}

Die Ergebnisse dieses Projektes sind sehr vielfältig. In diesem Beitrag werden Ergebnisse aus dem Bereich der Klinikversorgung vorgestellt. Resultate weiterer Kategorien werden in weiteren Publikationen veröffentlicht. Die Erfahrungen der Mitarbeiter und Angehörigen von Menschen mit geistigen und mehrfachen Behinderungen in der akut-stationären Versorgung sind dominierend von einem „Mangel“ gekennzeichnet. Die dargestellten Ergebnisse beziehen sich auf Erfahrungen der Interviewten in 5 Kliniken der Region, die jeweils von unterschiedlichen Trägern betrieben werden.

Mangel an Zeit und Personal I Die Aussagen zu Zeit und Personal wurden von den Interviewten 
häufig in einem Zusammenhang gemacht. Es wurden in den Aussagen Bilder formuliert wie „sind im Laufschritt unterwegs“, ,,sind im Stress“, „sind überlastet“, „können Personelles nicht leisten“ oder „sind auf Pflege nicht eingestellt.“

In den Interviews wurde beschrieben, dass die Mitarbeiter sich wegen des Personalmangels bereits bei der Aufnahme keine Zeit nehmen, um sich ein umfassendes Bild über die Situation des Erkrankten zu machen und vorab von Eltern oder Einrichtungen erstellte Berichte mit Informationen über bisherige Erkrankungen u. Ä. zu lesen.

Nicht angemessene pflegerische und medizinische Versorgung I Einige Interviewte stellten fest, dass die Personen mit Behinderung im Krankenhaus aus ihrer Perspektive nicht die angemessene pflegerische und medizinische Versorgung erhalten. Die mangelnde Zeit führte nach Ansicht von mehreren Befragten zu der Problematik, dass die Patienten nicht ausreichend Zeit für die Nahrungs- und Flüssigkeitsaufnahme haben. Es wird des Weiteren berichtet von

- abgelehnten Aufnahmen in einem Krankenhaus,

- nicht gewährten Therapien,

- nicht erkannter Oberschenkelhalsfraktur,

- Sedierungen und Fixierungen,

- nicht durchgeführten grundpflegerischen Leistungen (z.B. Hilfeleistungen bei Toilettengängen oder in der Körperhygiene).

Einige Mitarbeiter und Angehörige führten aus, dass sie häufig diese Maßnahmen in den Krankenhäusern übernehmen oder sich durch intensives Einbringen bei Ärzten und Pflegekräften erst Gehör verschaffen, um die erforderlichen oder aus ihrer Sicht angemessenen Therapien zu erreichen.

Eine Mitarbeiterin einer Einrichtung berichtete von einer Bewohnerin der Einrichtung, die vor der Krankenhausaufnahme kontinent war und in der Lage war, die Toilette aufzusuchen, jedoch im Krankenhaus eine Inkontinenzhose erhielt und schnell muskulär abbaute, da sie bei den Toilettengängen nicht mehr unterstützt wurde.

Laut einer Mutter führte eine nicht angemessene ärztliche Versorgung zu einem tragischen Vorfall. Ihre Tochter, die im Krankenhaus behandelt werden sollte, erhielt nicht die erforderliche Therapie: „Und die letzte Erfahrung, die ich gemacht habe mit ihr, war eine ganz schlechte. Sie ist auch daran gestorben. Es war hier im Krankenhaus. Sie sammelte immer Flüssigkeit im Magen, die nicht abfließen konnte. Das war im Krankenhaus auch bekannt und dann riefen mich die Mitarbeiter morgens an, dass ihr Bauch angeschwollen ist. Ich bin dann ins Krankenhaus gefahren und da lag sie dann schon auf der Liege und der Arzt schwirrte überall rum, aber nicht bei ihr. Und dann habe ich gefragt, ob er denn nicht eben eine Sonde legen könnte, damit der Druck weg ist vom Magen. Und dann sagte er zu mir, ja, wenn sie eine haben ... So, ich war völlig perplex und dann ist der Magen geplatzt und sie ist daran gestorben.“

Mangel an Erfahrungen und Qualifikation I Ein Mangel an Erfahrungen des Krankenhauspersonals im Umgang mit Menschen mit Behinderungen wurde von 12 Interviewten vermutet. Es wurde die Annahme formuliert, dass das ärztliche und pflegerische Personal überwiegend hilflos und sowohl mit Angst als auch Distanz agiere sowie wenig Fachwissen habe, da es zu wenig auf diese Zielgruppe in Studium und Ausbildung vorbereitet werde. Einige Interviewte machen vor diesem Hintergrund eine Überforderung des Personals aus.

Mangel an Kommunikation und Kooperation I Die Kommunikation und Kooperation zwischen Mitarbeitern der Wohneinrichtungen und Eltern sowie den Betroffenen und Mitarbeitern im Krankenhaus wurde in einigen Interviews als defizitär beschrieben. Einige Eltern berichteten, dass sie bei Tipps oder Hinweisen an das Pflegepersonal „teilweise Blicke von der Seite“ bekommen haben, oder dass die Kommunikation wegen sprachlicher Barrieren seitens der Ärzte mit Migrationshintergrund teilweise schwierig war, und dass sie sich als Eltern auf den Stationen nicht willkommen gefühlt haben.

In einem Interview einer Mitarbeiterin erscheint es, dass trotz des Bemühens, alle Informationen zu übergeben, die Kommunikation mit dem Krankenhaus nicht ganz gelang: „Wenn jemand ins Krankenhaus muss, begleiten wir natürlich immer die Fahrt dorthin, geben auch alles ab, was wir haben an Unterlagen. So, und dann fahren wir ja irgendwann und dann fängt eigentlich immer so ein bisschen, ab und zu, nicht immer, aber das Negative an. Dann heißt es so, sie haben keinen Verlegungsbericht mitbekommen oder kein Medikamentenblatt ... das ist dann ein bisschen schwierig ... Eigentlich ist unsere Nummer immer da. Sie können uns jederzeit anrufen, aber das tun sie dann auch teilweise nicht.“

Ganz offensichtlich werden Wohneinrichtungen von den Mitarbeitern der Kliniken nicht als Partner in der Gesundheitsversorgung wahrgenommen. Eine Mitarbeiterin berichtet von einer Verlegungssituation eines Bewohners, in der der Verlegungsbericht und die Angaben über die erforderliche Inkontinenz- und Sondenversorgung im Krankenhaus nicht aufgenommen wurden. In einem Fall wurde über eine plötzliche Entlassung eines Bewohners aus dem Krankenhaus wieder in die Wohneinrichtung berichtet. Das Krankenhaus hatte über eine anstehende Entlassung nicht informiert und die weitere Behandlung eines operierten Zehs ohne weitere Information bezüglich der Therapie und Wundversorgung der Einrichtung überlassen, die sich die Informationen mühsam einholen oder über selbst recherchieren musste. 
Mangel an Informationen - Mangel an Kontinuität | Einige Interviewte wunderten sich, dass eigens für mögliche Klinikaufenthalte erstellte Informationsmappen über die Person mit Behinderung offensichtlich nicht zur Kenntnis genommen wurden oder Fragen der Anamnese immer wieder neu bei jedem Krankenhausaufenthalt beantwortet werden mussten. Ein interviewtes Elternpaar hat diese Erfahrungen mehrfach in mehreren Kliniken gemacht, obwohl sie eine Informationsmappe zur Situation des Sohnes angefertigt haben, die dem Krankenhauspersonal zur Verfügung gestellt wird: „Wir bringen ja schon immer die Berichte mit. Alles so übersichtlich wie möglich in stichwortartigem Stil, also, was nun wichtig ist für ihn. Aber die lesen sich das gar nicht durch. Immer wieder kommen neue Leute, fragen immer dasselbe oder wissen überhaupt nichts ..."

Der Schichtwechsel scheint eine weitere Problematik zu sein, die die Kontinuität in der Versorgung reduziert. Es entstand der Eindruck, dass die nachfolgende Schicht keine Informationen über den Patienten und seiner Behinderung sowie den Verlauf der Therapie und Versorgung hat.

Erwartungen und Verständnis Krankenhaus von und über Wohneinrichtungen I Einige Befragte äußerten die Vermutung, dass die Mitarbeiter in Krankenhäusern Erwartungen an die Wohneinrichtungen stellen, die diese nicht halten können, da sie keine Einrichtung der gesundheitlichen und pflegerischen Versorgung sind. Demgemäß wurde in einem Interview geäußert, dass die Krankenhäuser erwarten, dass die Mitarbeiter der Einrichtungen die Bewohner in die Krankenhäuser begleiten. Manchmal beabsichtigen die Krankenhäuser Bewohner wieder in die Einrichtung zu entlassen, obwohl zunächst eine Rehabilitation anstünde. In die Einrichtung von einer interviewten Mitarbeiterin sollte auch ein Bewohner mit gesundheitlichem und pflegerischem Bedarf entlassen werden. Die Mitarbeiterin führte aus, dass sie dem Krankenhaus verdeutlichen musste, dass sie den Bedarf nicht decken können, da es sich bei dieser Einrichtung um eine Wohneinrichtung handele, die weder ausreichend noch angemessen auf die gesundheitliche Versorgung des Bewohners eingerichtet sei noch über dafür qualifiziertes Personal verfüge.

\section{Diskussion}

Die Versorgung von Menschen mit geistigen und mehrfachen Behinderungen im Krankenhaus wird nicht nur in dieser Studie, sondern auch in einigen anderen Publikationen als defizitär beschrieben [6, 25, 26, 27], vor allen in den Bereichen

- Zeit,

- Qualifikation,

- Kompetenzen und

- Personalausstattung.
Die Mangelerfahrungen hinsichtlich Personal und Zeit sind in einem Zusammenhang zu sehen. Es ist nachvollziehbar, dass der von den Befragten mehrfach geäußerte und „gefühlte“ Personalmangel in den Krankenhäusern dazu führt, dass das Krankenhauspersonal weniger Zeit für die Zielgruppe Menschen mit Behinderungen hat. Mehrere Veröffentlichungen belegen, dass in den Jahren von 1996-2008 umfangreiche Kürzungen beim Pflegepersonal im Umfang von 50000 Vollzeitstellen vorgenommen wurden [18, 24]. Zwar ist seit dem Jahr 2005 zu erkennen, dass dieser Trend gestoppt wurde. Im Jahr 2008 konnten bundesweit bereits wieder 1840 Vollzeitstellen in den Kliniken aufgebaut werden [18].

Jedoch ist fraglich, ob diese positive Tendenz anhaltend ist und den erhöhten Anforderungen in der Krankenhausversorgung gerecht wird, da insgesamt die Fallzahl in den Krankenhäusern in den letzten Jahren gestiegen sowie die Liegezeit der Patienten gesunken ist, so dass die Kliniken insgesamt eine höhere Arbeitsdichte verspüren $[4,18]$. Problematisch ist zu beurteilen, dass mit der Kürzung der Verweildauer „eine Intensivierung der Therapie einhergeht“ [18]. In der Wahrnehmung der Pflegekräfte resultieren aus dieser Situation Mängel in der Patientenversorgung im Krankenhaus, da beispielsweise Patienten weniger

- überwacht (monitoring),

- mobilisiert,

- angeleitet,

- geschult,

- in der Ernährung, Grundpflege und emotional und bei Toilettengängen weniger unterstützt,

- gelagert

- kommuniziert oder

- Medikamente fachlich weniger angemessen verabreicht werden $[2,31,20]$.

Gerade diese Maßnahmen stellen jedoch die Bedarfe in der Versorgung von Menschen mit Behinderungen in Krankenhäusern dar [31, 11, 5].

Demgemäß kann die Hypothese formuliert werden, dass der subjektive Eindruck der Interviewten die Situation der Versorgung in den Krankenhäusern widerspiegelt. Sehr wahrscheinlich sind Menschen mit Behinderungen ohne eine Unterstützung von Mitarbeitern der Wohneinrichtungen oder deren Angehörigen in den Krankenhäusern in wesentlichen grundpflegerischen und therapeutischen Bereichen unterversorgt. Ihre Bedarfe werden vermutlich deswegen nicht erfüllt, weil in der Versorgung im Krankenhaus zusätzlich zu den Bedarfen aus der akuten Erkrankung noch die behinderungsspezifischen Bedarfe und Bedürfnisse hinzukommen [6, 31]. Dieser Mehraufwand spiegelt sich jedoch nicht in der Vergütung der Krankenhäuser für Menschen mit geistigen und mehrfachen Behinderungen wider, so dass die Kosten- und Personalaufwände nicht entsprechend refinanziert werden [15]. Für Personen mit Behinderungen im Krankenhaus be- 
steht die Gefahr, dass aufgrund einer nicht angemessenen Versorgung sich die behinderungsspezifischen Einschränkungen während des Krankenhausaufenthaltes verstärken können, „wenn neben dem durch die Krankheit hervorgerufenen Unterstützungsbedarf der alltägliche und individuenspezifische Bedarf nicht wahrgenommen wird“" [6].

Die vereinzelt geschilderten Erfahrungen, dass eine Behandlung im Krankenhaus nicht gewährt wurde, können unter Umständen mit den Entgeltsystemen oder personellen sowie fachlichen Ressourcen der Krankenhäuser in einem Zusammenhang stehen. Es liegen aber keine systematischen Erkenntnisse vor, dass Menschen mit Behinderungen weniger häufig notwendige Behandlungen in einem Krankenhaus erhalten, so dass nicht ausgeschlossen werden kann, dass Einzelfälle davon betroffen sind. Dessen ungeachtet erscheint eine systematische Untersuchung zur Frage des Zugangs zur Krankenhausversorgung und geistiger oder mehrfacher Behinderung von großer Relevanz, wenn diese Bevölkerungsgruppe als gefährdet eingeschätzt wird und bestimmte Ungleichheiten in der gesundheitlichen Versorgung angenommen werden können. Es ist anzunehmen, dass die Rechtzeitigkeit von Krankenhausbehandlungen gerade bei Menschen mit Behinderungen und erhöhten Risiken gesundheitlicher Einschränkungen und Verschlechterungen bedeutsam ist.

Der Mangel an Information und an Kontinuität in der personellen Situation wird von vielen Befragten in der Krankenhausversorgung geschildert. Die in Krankenhäusern üblichen Schichtwechsel erhöhen die Problematik, da immer wieder neue Personen die gesundheitliche und pflegerische Versorgung übernehmen und offensichtlich nicht umfassend über die Patienten mit Behinderung informiert werden [31]. Probleme dieser Art sind der Krankenhausversorgung inhärent und gelten auch für Menschen ohne Behinderungen. Jedoch können aufgrund der Gefährdung dieser Personengruppe [17] die Auswirkungen noch nicht abgeschätzt werden.

Ein weiterer Mangel wird in der fehlenden Qualifikation oder in der geringen Erfahrung von Ärzten und Pflegekräften sowohl im niedergelassenen Bereich als auch in den Krankenhäusern betrachtet. Dieser Mangel kann die von den Interviewten beobachtete Unsicherheit im Umgang mit Menschen mit Behinderungen begründen. Analysen aus dem internationalen und nationalen Raum zur Qualifikation des medizinischen Personals für die gesundheitliche und pflegerische Versorgung von Menschen mit geistigen und mehrfachen Behinderungen unterstützen den Eindruck einer nicht ausreichenden Ausbildung [16, 19, 29, 31].

Der Mangel an Kommunikation und Kooperation mit und zwischen den Sektoren, Mitarbeitern so- wie Krankenhäusern resultiert u. a. in unvorbereiteten Entlassungen aus dem Krankenhaus. Die Angehörigen und / oder Mitarbeiter erhalten vielfach keine oder zu wenige Informationen über den Therapieverlauf im Krankenhaus, erforderliche Therapien oder erforderliche Hilfsmittel. Da Mitarbeiter und Eltern häufig als Fürsprecher und Dolmetscher sowohl für das medizinische Personal sowie für die Menschen mit Behinderungen in der gesundheitlichen Versorgung agieren, ist die geschilderte schwierige Kommunikation mit Ärzten als problematisch zu bewerten. Es besteht die Gefahr, dass wichtige Informationen für beide Seiten verloren gehen und nicht in eine adäquate $\mathrm{Di}$ agnostik oder Bewertung von anstehenden therapeutischen Maßnahmen eingehen. Da die Mitarbeiter und/oder Eltern als Advokaten fungieren [29], ist eine effektive Kommunikation mit Ärzten und anderem medizinischen Personal von hoher Bedeutung für eine gute Gesundheitsversorgung.

Inwiefern eine defizitäre Kommunikation und Kooperation zwischen Betreuern und medizinischem Personal als Benachteiligung für Menschen mit Behinderungen angenommen werden muss und wie sich diese auf den Gesundheitszustand auswirkt, ist noch fraglich. Sie stellt jedoch eine Barriere in der gesundheitlichen Versorgung von Menschen mit geistigen Behinderungen dar [10]. Es ist möglich, dass die von den Interviewten vermuteten geringen Erfahrungen und wahrgenommene nicht ausreichende Qualifikation des medizinischen Personals für die gesundheitliche Versorgung der Menschen mit geistigen und mehrfachen Behinderungen diese kommunikative Barriere begünstigen [10, 31].

Aus den Interviews kann der Eindruck entstehen, dass medizinisches Personal, das in Kliniken arbeitet, ein wenig differenziertes Verständnis von Wohneinrichtungen haben, in denen Menschen mit Behinderungen leben [31]. Dies führt u.a. dazu, dass Menschen mit weiterem Therapiebedarf früh aus dem Krankenhaus entlassen werden und die Mitarbeiter erklären müssen, dass die Wohneinrichtung keine Pflegeeinrichtung sei und den gesundheitlichen Bedarf nicht decken kann [31].

Es kommt auch vor, dass Mitarbeiter von Wohneinrichtungen im Krankenhaus oder bei behandelnden Ärzten sich Informationen über weitere Therapien und Maßnahmen beschaffen müssen, da diese nicht kommuniziert werden [31]. Die Literaturlage legt nahe, dass die Erfahrungen während eines Krankenhausaufenthaltes für Menschen mit Behinderungen von nachhaltiger Wirkung sind $[6,8,12]$. Vorherige negative Erfahrungen können dazu führen, dass der Mensch mit Behinderung diese auf zukünftige Krankenhausaufenthalte überträgt und eine Überweisung verweigert [12]. Vor diesem Hintergrund ist empfehlenswert, die Versorgung von Menschen mit 
Behinderungen in Krankenhäusern kritisch zu reflektieren und angemessene Konzepte, Maßnahmen und Interventionen bzw. Regelwerke zu entwickeln.

\section{Konsequenzen für Klinik und Praxis}

- Aus der Perspektive von Betreuern und Angehörigen von Menschen mit geistigen und mehrfachen Behinderungen ist die Versorgung im Krankenhaus defizitär.

- Es werden Maßnahmen, Konzepte und Interventionen in den Kliniken benötigt, um eine qualitativ hochwertige Gesundheitsversorgung dieser gefährdeten Bevölkerungsgruppe zu ermöglichen.

- Es bedarf einer angemessenen Qualifikation des an der Gesundheitsversorgung beteiligten medizinischen Personals für Menschen mit Behinderungen im Krankenhaus.

\section{Literatur}

1 Bogner A, Menz W. Experteninterviews in der qualitativen Sozialforschung. Zur Einführung in eine sich intensivierende Methodendebatte. Experteninterviews. In: Bogner A, Littig B, Menz W, Hrsg. Experteninterviews - Theorien. Methoden, Anwendungsfelder. Wiesbaden: Verlag für Sozialwissenschaften; 2009: 7-31

2 Böhm A. Theoretisches Codieren: Textanalyse in der Grounded Theory. In: Flick, $U$ et al., Hrsg. Qualitative Forschung Ein Handbuch. Berlin: Rowolth; 2004: 475-485

3 Bradbury-Jones C, Rattray J, Jones M, Macgillivray S. Promoting the health, safety and welfare of adults with learning disabilties in the acute setting: a structured literature review. J Clin Nurs 2013; 22; 1497-1509

4 Braun B, Müller R, Timm A. Gesundheitliche Belastungen, Arbeitsbedingungen und Erwerbsbiographien von Pflegekräften im Krankenhaus. Eine Untersuchung vor dem Hintergrund der DRG-Einführung. In: GEK, Gmünder ErsatzKasse, Hrsg. Schriftenreihe zur Gesundheitsanalyse/32. Hippe: Asgard; 2004

5 Brühl P. Gesundheitsversorgung bei Menschen mit geistiger Behinderung. Patienten-Beschwerde-und Beratungsstelle des UKB 2009 http://www.lveb-nrw.de/Prof_Bruehl_ Gesundheitsversorgung.pdf Letzter Zugriff am 15.09.15

6 Budroni H. Pflegeerfahrungen körperbehinderter Menschen im Krankenhaus. Die Schwester/Der Pfleger 2006; 45: 458-462

7 Corbin J. Die Methode der Grounded Theory im Überblick. In: Schaeffer D, Müller-Mundt G, Hrsg. Qualitative Gesundheits- und Pflegeforschung. Göttingen: Hans Huber; 2002: 59-70

8 Cumella S, Martin D. Secondary healthcare and learning disability. J Intellect Disabil 2004; 8: 30-40

9 Cutcliffe JR. Methodological issues in grounded theory. J Adv Nurs 2000; 31: 1476-1484

10 Ding-Greiner C, Kruse A, Hrsg. Betreuung und Pflege geistig behinderter und chronisch kranker Menschen im Alter. Beiträge aus der Praxis. Stuttgart: Kohlhammer; 2010

11 Gaskell S, Nightingale S. Supporting people with learning disabilities in acute care. Nurs Stand 2010; 24: 42-48
12 Gibbs SM, Brown MJ, Muir WJ. The experiences of adults with intellectual disabilities and their careers in general hospitals: a focus group study. J Intellect Disabil Res 2008; 52: 1061-1077

13 Glaser BG, Strauss AL. Grounded Theory. Strategien qualitativer Forschung. Bern: Huber; 2010

14 Gläser ], Laudel G. Experteninterviews und qualitative Inhaltsanalyse. 4. Aufl. Wiesbaden: Verlag für Sozialwissenschaften; 2010

15 Harenski K. Alles andere als Wunschpatienten. Dtsch Ärztebl 2007; 27: 1970-1971

16 Hasseler M. Prävention und Gesundheitsförderung in der Pflege - ein konzeptioneller Ansatz. Weinheim: Beltz Juventa; 2011

17 Hasseler M. Menschen mit Behinderungen als vulnerable Bevölkerungsgruppe in gesundheitlicher und pflegerischer Versorgung [Habilitationsschrift]. Ostfalia Hochschule für angewandte Wissenschaften, Wolfsburg; 2014

18 Isfort M, Weidner F. Der Pflegemangel im Krankenhaus wird chronisch. Die Schwester/Der Pfleger 2010; 40: 1-9

19 Jenkins R. The role of nurses in meeting the health care needs of older people with intellectual disabilities: A review of the published literature. J Intellect Disabil 2012; 16: 85-95

20 Kalisch B], Tschannen D, Lee H, Friese CR. Hospital variation in missed nursing care. Am J Med Qual 2011; 26: 291-299

21 McMillan Boyles C, Bailey PH, Mossey S. Representations of disability in nursing and healthcare: an integrative review. J Adv Nurs 2008; 62: 428-437

22 Meuser M, Nagel U. Experteninterviews und der Wandel der Wissensproduktion. In: Bogner A, Littig B, Menz W, Hrsg. Experteninterviews - Theorien. Methoden, Anwendungsfelder. Wiesbaden: Verlag für Sozialwissenschaften; 2009: 35-60

23 Müller-Mundt G. Experteninterviews oder die Kunst der Entlockung „funktionaler Erzählungen“. In: Schaeffer D, Müller-Mundt G, Hrsg.Qualitative Gesundheits- und Pflegeforschung. Göttingen: Hans Huber; 2002: 269-283

24 Deutsches Institut für angewandte Pflegeforschung e. V. Pflege-Thermometer 2009. http://www.dip.de/fileadmin/data/pdf/material/ dip_Pflege-Thermometer_2009.pdf Letzter Zugriff am 15.09.15

25 Sandforth E, Hasseler M. Gesundheitsförderung in stationären Wohneinrichtungen für Menschen mit Mehrfachbehinderung. Pflege \& Gesellschaft 2014; 19; 167-186

26 Schäper S, Schüller S, Dieckmann F, Greving H. Anforderungen an die Lebensgestaltung älter werdender Menschen mit geistiger Behinderung in unterstützten Wohnformen - Ergebnisse einer Literaturanalyse und Expertenbefragung. Zwischenbericht zum Forschungsprojekt „Lebensqualität inklusiv(e): Innovative Konzepte unterstützten Wohnens älter werdender Menschen mit Behinderung" Katholische Hochschule NRW 2010 http://www.lwl.org/@@afiles/28598234/ zweiter_zwischenbericht.pdf Letzter Zugriff am 15.09.15

27 Seidel M. Die Situation von Patientinnen und Patienten mit geistiger und mehrfacher Behinderung im Krankenhaus - ein Problemaufriss. In: Bundesverband evangelische Behindertenhilfe e. V. Patientinnen und Patienten mit geistiger und mehrfacher Behinderung im Krankenhaus - Problemlagen und Lösungsperspektiven. Dokumentation des Symposiums am 4. Februar 2010 http://www.mara.de/fileadmin/Krankenhaus_ Mara/downloads/Dokumentation Symposium_020810.pdf Letzter Zugriff am 15.09.15 
28 Smeltzer SC, Avery C, Haynor P. Interactions of people with disabilities and nursing staff during hospitalization. Am J Nurs 2012; 112: 30-37

29 Steffen P, Blum K. Menschen mit geistiger Behinderung: Defizite in der Versorgung. Dtsch Ärzteblatt 2012; 109; 17: A-860-A-862

30 Taua C, Hepworth J, Neville C. Nurses' role in caring for people with a comorbidity of mental illness and intellectual disability: A literature review. Int J Ment Health Nurs 2012; 21: 163-174

31 Truschkat I, Kaiser-Belz M, Volkmann V. Theoretisches Sampling in Qualifikationsarbeiten. Die Grounded Theory Methodologie zwischen Programmatik und Forschungspraxis. In: Mey G, Mruck K, Hrsg. Grounded Theory Reader. Wiesbaden: Verlag für Sozialwissenschaften; 2011: 353-380

32 Webber R, Bowers B, Bigby C. Hospital experiences of older people with intellectual disability: Responses of group home staff and family members. J Intellect Dev Disabil 2010; 35: 155-164

Interessenkonflikt

Die Autorin gibt an, dass kein Interessenkonflikt besteht.

DOI 10.1055/s-0041-106610 Dtsch Med Wochenschr 2015; 140: e217-e223

(c) Georg Thieme Verlag KG . Stuttgart - New York . ISSN 0012-0472 